TAIWANESE JOURNAL OF MATHEMATICS

Vol. 4, No. 3, pp. 417-426, September 2000

\title{
A CERTAIN FAMILY OF FRACTIONAL DIFFERINTEGRAL EQUATIONS
}

\author{
Shih-Tong Tu, Yu-Tan Huang, I-Chun Chen and H. M. Srivastava
}

\begin{abstract}
In recent years, several workers demonstrated the usefulness of fractional calculus in the derivation of particular solutions of a number of familiar second-order differential equations associated (for example) with Gauss, Legendre, Jacobi, Chebyshev, Coulomb, Whittaker, Euler, Hermite, and Weber equations. The main object of this paper is to show how some of the most recent contributions on this subject, involving the Weber equations and their various generalized forms, can be obtained by suitably applying a general theorem on particular solutions of a certain family of fractional differintegral equations.
\end{abstract}

\section{Introduction, Definitions, And Preliminaries}

The subject of fractional calculus (that is, derivatives and integrals of any real or complex order) has gained importance and popularity during the past three decades or so, due mainly to its demonstrated applications in numerous seemingly diverse fields of science and engineering (see, for details, [3]). By applying the following definition of a fractional differintegral (that is, fractional derivative or fractional integral) of order $\nu \in \mathbb{R}$, many authors have derived particular solutions of a number of families of homogeneous (as well as nonhomogeneous) linear fractional differintegral equations.

Definition (cf. $[1,2,4])$. If the function $f(z)$ is analytic and has no branch point inside and on $\mathcal{C}$, where

$$
\mathcal{C}:=\left\{\mathcal{C}^{-}, \mathcal{C}^{+}\right\}
$$

Communicated by P. Y. Wu.

Received March 7, 2000.

2000 Mathematics Subject Classification: Primary 26A33, 34A05; Secondary 34A25.

Key words and phrases: Fractional calculus, differintegral equation, Weber equation, generalized Leibniz rule, analytic function, integral curve. 
$\mathcal{C}^{-}$is an integral curve along the cut joining the points $z$ and $-\infty+i \Im(z), \mathcal{C}^{+}$ is an integral curve along the cut joining the points $z$ and $\infty+i \mathfrak{I}(z)$,

$$
\begin{gathered}
f_{\nu}(z)={ }_{\mathcal{C}} f_{\nu}(z):=\frac{\Gamma(\nu+1)}{2 \pi i} \int_{\mathcal{C}} \frac{f(\zeta) d \zeta}{(\zeta-z)^{\nu+1}} \\
\left(\nu \in \mathbb{R} \backslash \mathbb{Z}^{-} ; \mathbb{Z}^{-}:=\{-1,-2,-3, \ldots\}\right)
\end{gathered}
$$

and

$$
f_{-n}(z):=\lim _{\nu \rightarrow-n}\left\{f_{\nu}(z)\right\} \quad(n \in \mathbb{N}:=\{1,2,3, \ldots\})
$$

where $\zeta \neq z$,

$$
-\pi \leqq \arg (\zeta-z) \leqq \pi \quad \text { for } \quad \mathcal{C}^{-}
$$

and

$$
0 \leqq \arg (\zeta-z) \leqq 2 \pi \quad \text { for } \quad \mathcal{C}^{+}
$$

then $f_{\nu}(z)(\nu>0)$ is said to be the fractional derivative of $f(z)$ of order $\nu$ and $f_{\nu}(z)(\nu<0)$ is said to be the fractional integral of $f(z)$ of order $-\nu$, provided that

$$
\left|f_{\nu}(z)\right|<\infty \quad(\nu \in \mathbb{R})
$$

Remark 1. Throughout the present work, we shall simply write $f_{\nu}$ for $f_{\nu}(z)$ whenever the argument of the differintegrated function $f$ is clearly understood by the surrounding context. Moreover, in case $f$ is a many-valued function, we shall tacitly consider the principal value of $f$ in our investigation. For the sake of convenience in dealing with their various (known or new) special cases, we choose also to state each of the fundamental results (Theorems 1 and 2 below) for fractional differintegral equations of a general order $\mu \in \mathbb{R}$.

We find it to be worthwhile to recall here the following useful lemmas and properties associated with the fractional differintegration which is defined above (cf., e.g., [1, 2]).

Lemma 1 (Linearity Property). If the functions $f(z)$ and $g(z)$ are singlevalued and analytic in some domain $\Omega \subseteq \mathbb{C}$, then

$$
\left(k_{1} f+k_{2} g\right)_{\nu}=k_{1} f_{\nu}+k_{2} g_{\nu} \quad(\nu \in \mathbb{R} ; z \in \Omega)
$$

for any constants $k_{1}$ and $k_{2}$. 
Lemma 2 (Index Law). If the function $f(z)$ is single-valued and analytic in some domain $\Omega \subseteq \mathbb{C}$, then

$$
\begin{gathered}
\left(f_{\mu}\right)_{\nu}=f_{\mu+\nu}=\left(f_{\nu}\right)_{\mu} \\
\left(f_{\mu} \neq 0 ; \quad f_{\nu} \neq 0 ; \quad \mu, \nu \in \mathbb{R} ; \quad z \in \Omega\right) .
\end{gathered}
$$

Lemma 3 (Generalized Leibniz Rule). If the functions $f(z)$ and $g(z)$ are single-valued and analytic in some domain $\Omega \subseteq \mathbb{C}$, then

$$
(f \cdot g)_{\nu}=\sum_{n=0}^{\infty}\left(\begin{array}{l}
\nu \\
n
\end{array}\right) f_{\nu-n} \cdot g_{n} \quad(\nu \in \mathbb{R} ; z \in \Omega),
$$

where $g_{n}$ is the ordinary derivative of $g(z)$ of order $n\left(n \in \mathbb{N}_{0}:=\mathbb{N} \cup\{0\}\right)$, it being tacitly assumed (for simplicity) that $g(z)$ is the polynomial part (if any) of the product $f(z) g(z)$.

Property 1. For a constant $\lambda$,

$$
\left(e^{\lambda z}\right)_{\nu}=\lambda^{\nu} e^{\lambda z} \quad(\lambda \neq 0 ; \nu \in \mathbb{R} ; z \in \mathbb{C}) .
$$

Property 2. For a constant $\lambda$,

$$
\left(e^{-\lambda z}\right)_{\nu}=e^{-i \pi \nu} \lambda^{\nu} e^{-\lambda z} \quad(\lambda \neq 0 ; \nu \in \mathbb{R} ; z \in \mathbb{C}) .
$$

Property 3. For a constant $\lambda$,

$$
\begin{gathered}
\left(z^{\lambda}\right)_{\nu}=e^{-i \pi \nu} \frac{\Gamma(\nu-\lambda)}{\Gamma(-\lambda)} z^{\lambda-\nu} \\
(\nu \in \mathbb{R} ; z \in \mathbb{C} ;|\Gamma(\nu-\lambda) / \Gamma(-\lambda)|<\infty) .
\end{gathered}
$$

Some of the most recent contributions on the subject of particular solutions of fractional differintegral equations are those by Tu et al. [5], who considered some generalizations of the classical Weber equations. We recall here the main results of $\mathrm{Tu}$ et al. [5] as Theorems A and B below.

Theorem A (cf. [5, Theorems 1.1 and 1.3]). If the given function $f$ satisfies the constraint (1.6) and $f_{\frac{1}{2}(\lambda-1)} \neq 0$, then the generalized nonhomogeneous Weber equation:

$$
\sum_{k=0}^{n}\left(\begin{array}{c}
\frac{1}{2}(1-\lambda) \\
k
\end{array}\right)\left[\left(z^{n}\right)_{k} \phi_{m-k}(z)-2\left(z^{n+1}\right)_{k} \phi_{m-k-1}(z)\right]
$$




$$
\begin{gathered}
-2\left(\begin{array}{c}
\frac{1}{2}(1-\lambda) \\
n+1
\end{array}\right)\left(z^{n+1}\right)_{n+1} \phi_{m-n-2}(z)=f(z) e^{\frac{1}{2} z^{2}} \\
\left(m \in \mathbb{Z}:=\mathbb{N}_{0} \cup \mathbb{Z}^{-} ; n \in \mathbb{N}_{0} ; z \in \mathbb{C}\right)
\end{gathered}
$$

has a particular solution of the form:

$$
\phi(z)=\left(\left(\left(f(z) \cdot e^{\frac{1}{2} z^{2}}\right)_{\frac{1}{2}(\lambda-1)} \cdot z^{-n} \cdot e^{-z^{2}}\right)_{-1} \cdot e^{z^{2}}\right)_{\frac{1}{2}(1-\lambda)-m+1},
$$

provided that the second member of (1.14) exists, $\lambda$ being a given constant.

Furthermore, the generalized homogeneous Weber equation:

$$
\begin{gathered}
\sum_{k=0}^{n}\left(\begin{array}{c}
\frac{1}{2}(1-\lambda) \\
k
\end{array}\right)\left[\left(z^{n}\right)_{k} \phi_{m-k}(z)-2\left(z^{n+1}\right)_{k} \phi_{m-k-1}(z)\right] \\
-2\left(\begin{array}{c}
\frac{1}{2}(1-\lambda) \\
n+1
\end{array}\right)\left(z^{n+1}\right)_{n+1} \phi_{m-n-2}(z)=0 \\
\left(m \in \mathbb{Z} ; n \in \mathbb{N}_{0} ; z \in \mathbb{C}\right)
\end{gathered}
$$

has solutions of the form:

$$
\phi(z)=K\left(e^{z^{2}}\right)_{\frac{1}{2}(1-\lambda)-m+1},
$$

where $K$ is an arbitrary constant, $\lambda$ is a given constant, and the second member of (1.16) is assumed to exist.

Theorem B (cf. [5, Theorems 1.2 and 1.3]). If the given function $f$ satisfies the constraint (1.6) and $f_{\frac{1}{2}(\lambda-1)} \neq 0$, then the generalized nonhomogeneous Weber equation:

$$
\begin{gathered}
\sum_{k=0}^{n}\left(\begin{array}{c}
\frac{1}{2}(1-\lambda) \\
k
\end{array}\right)\left[\left(z^{n}\right)_{k}\left(\phi(z) e^{\frac{1}{2} z^{2}}\right)_{m-k}-2\left(z^{n+1}\right)_{k}\left(\phi(z) e^{\frac{1}{2} z^{2}}\right)_{m-k-1}\right] \\
-2\left(\begin{array}{c}
\frac{1}{2}(1-\lambda) \\
n+1
\end{array}\right)\left(z^{n+1}\right)_{n+1}\left(\phi(z) e^{\frac{1}{2} z^{2}}\right)_{m-n-2}=f(z) e^{\frac{1}{2} z^{2}} \\
\left(m \in \mathbb{Z} ; n \in \mathbb{N}_{0} ; z \in \mathbb{C}\right)
\end{gathered}
$$

has a particular solution of the form:

$$
\phi(z)=e^{-\frac{1}{2} z^{2}}\left(\left(\left(f(z) \cdot e^{\frac{1}{2} z^{2}}\right)_{\frac{1}{2}(\lambda-1)} \cdot z^{-n} \cdot e^{-z^{2}}\right)_{-1} \cdot e^{z^{2}}\right)_{\frac{1}{2}(1-\lambda)-m+1}
$$


provided that the second member of (1.18) exists, $\lambda$ being a given constant.

Furthermore, the generalized homogeneous Weber equation:

$$
\begin{gathered}
\sum_{k=0}^{n}\left(\begin{array}{c}
\frac{1}{2}(1-\lambda) \\
k
\end{array}\right)\left[\left(z^{n}\right)_{k}\left(\phi(z) e^{\frac{1}{2} z^{2}}\right)_{m-k}-2\left(z^{n+1}\right)_{k}\left(\phi(z) e^{\frac{1}{2} z^{2}}\right)_{m-k-1}\right] \\
-2\left(\begin{array}{c}
\frac{1}{2}(1-\lambda) \\
n+1
\end{array}\right)\left(z^{n+1}\right)_{n+1}\left(\phi(z) e^{\frac{1}{2} z^{2}}\right)_{m-n-2}=0 \\
\left(m \in \mathbb{Z} ; n \in \mathbb{N}_{0} ; z \in \mathbb{C}\right)
\end{gathered}
$$

has solutions of the form:

$$
\phi(z)=K e^{-\frac{1}{2} z^{2}}\left(e^{z^{2}}\right)_{\frac{1}{2}(1-\lambda)-m+1},
$$

where $K$ is an arbitrary constant, $\lambda$ is a given constant, and the second member of (1.20) is assumed to exist.

The assertions of Theorems A and B are substantially the same results stated with only simple notational changes. In fact, the assertions of Theorem B would follow immediately from those of Theorem A by merely making the following change of notations:

$$
\phi(z) \mapsto \phi(z) e^{\frac{1}{2} z^{2}} \quad(z \in \mathbb{C}) .
$$

Theorem A, on the other hand, would follow readily from Theorem B with $\phi(z)$ replaced trivially by $\phi(z) e^{-\frac{1}{2} z^{2}}$. In view of these observations, it would be sufficient to show here how Theorem A ( for example) can be derived by suitably applying a general theorem on particular solutions of a certain class of fractional differintegral equations.

\section{A General Theorem and Its Applications}

The following general theorem (due to Tu et al. [6]) provides a unification as well as generalization of a considerably large number of widely scattered results on the solutions of various families of homogeneous and nonhomogeneous fractional differintegral equations.

Theorem 1 (Tu et al. [6, Theorems 1 and 2]). Let $P(z ; p)$ and $Q(z ; q)$ be polynomials in $z$ of degrees $p$ and $q$, respectively, defined by

$$
\begin{aligned}
P(z ; p): & =\sum_{k=0}^{p} a_{k} z^{p-k} \\
& =a_{0} \prod_{j=1}^{p}\left(z-z_{j}\right) \quad\left(a_{0} \neq 0 ; p \in \mathbb{N}\right)
\end{aligned}
$$


and

$$
Q(z ; q):=\sum_{k=0}^{q} b_{k} z^{q-k} \quad\left(b_{0} \neq 0 ; q \in \mathbb{N}\right) .
$$

Suppose also that $f_{-\nu}(\neq 0)$ exists for a given function $f$.

Then the nonhomogeneous linear ordinary fractional differintegral equation:

$$
\begin{gathered}
P(z ; p) \phi_{\mu}(z)+\left[\sum_{k=1}^{p}\left(\begin{array}{l}
\nu \\
k
\end{array}\right) P_{k}(z ; p)+\sum_{k=1}^{q}\left(\begin{array}{c}
\nu \\
k-1
\end{array}\right) Q_{k-1}(z ; q)\right] \phi_{\mu-k}(z) \\
+\left(\begin{array}{c}
\nu \\
q
\end{array}\right) q ! b_{0} \phi_{\mu-q-1}(z)=f(z) \\
(\mu, \nu \in \mathbb{R} ; p, q \in \mathbb{N})
\end{gathered}
$$

has a particular solution of the form:

$$
\begin{gathered}
\phi(z)=\left(\left(\frac{f_{-\nu}(z)}{P(z ; p)} e^{H(z ; p, q)}\right)_{-1} e^{-H(z ; p, q)}\right)_{\nu-\mu+1} \\
\left(z \in \mathbb{C} \backslash\left\{z_{1}, \ldots, z_{p}\right\}\right),
\end{gathered}
$$

where, for convenience,

$$
H(z ; p, q):=\int^{z} \frac{Q(\zeta ; q)}{P(\zeta ; p)} d \zeta \quad\left(z \in \mathbb{C} \backslash\left\{z_{1}, \ldots, z_{p}\right\}\right)
$$

provided that the second member of (2.4) exists.

Furthermore, the homogeneous linear ordinary fractional differintegral equation:

$$
\begin{gathered}
P(z ; p) \phi_{\mu}(z)+\left[\sum_{k=1}^{p}\left(\begin{array}{l}
\nu \\
k
\end{array}\right) P_{k}(z ; p)+\sum_{k=1}^{q}\left(\begin{array}{c}
\nu \\
k-1
\end{array}\right) Q_{k-1}(z ; q)\right] \phi_{\mu-k}(z) \\
+\left(\begin{array}{c}
\nu \\
q
\end{array}\right) q ! b_{0} \phi_{\mu-q-1}(z)=0 \\
(\mu, \nu \in \mathbb{R} ; p, q \in \mathbb{N})
\end{gathered}
$$

has solutions of the form:

$$
\phi(z)=K\left(e^{-H(z ; p, q)}\right)_{\nu-\mu+1},
$$


where $K$ is an arbitrary constant and $H(z ; p, q)$ is given by (2.5), it being provided that the second member of (2.7) exists.

Remark 2. It should be remarked in passing that Tu et al. [6, Section 3] also gave the solutions of several general families of partial fractional differintegral equations analogous to (2.3) and (2.6). More importantly, as already observed in conclusion by $\mathrm{Tu}$ et al. [6], either or both of the polynomials $P(z ; p)$ and $Q(z ; q)$, involved in Theorem 1 , can be of degree 0 as well. Thus, in the definitions (2.1) and (2.2) (as also in Theorem 1 and its such consequences as Theorem 2 below), $\mathbb{N}$ may be replaced (if and where needed) by $\mathbb{N}_{0}$.

With a view to applying Theorem 1 , we first replace $q$ by $q+1$ and set

$$
a_{0}=\alpha(\alpha \neq 0) \quad \text { and } \quad a_{1}=\cdots=a_{p}=0
$$

and

$$
b_{0}=\beta(\beta \neq 0) \quad \text { and } \quad b_{1}=\cdots=b_{q+1}=0,
$$

so that

$$
P(z ; p)=\alpha z^{p} \quad(\alpha \neq 0 ; p \in \mathbb{N})
$$

and

$$
Q(z ; q+1)=\beta z^{q+1} \quad\left(\beta \neq 0 ; q \in \mathbb{N}_{0}\right) .
$$

We thus find from (2.5), (2.10), and (2.11) that

$$
H(z ; p, q+1)=\frac{\beta}{\alpha} \int^{z} t^{q-p+1} d t \quad\left(p \in \mathbb{N} ; q \in \mathbb{N}_{0}\right)
$$

or, equivalently, that

$$
H(z ; p, q+1)= \begin{cases}\frac{\beta z^{q-p+2}}{\alpha(q-p+2)} & (p \neq q+2) \\ \frac{\beta}{\alpha} \log z & (p=q+2) .\end{cases}
$$

A special case of Theorem 1 can now be stated as

Theorem 2. Suppose that $f_{-\nu}(\neq 0)$ exists for a given function $f$. Then the nonhomogeneous linear ordinary fractional differintegral equation:

$$
\alpha z^{p} \phi_{\mu}(z)+\left[\sum_{k=1}^{p}\left(\begin{array}{l}
\nu \\
k
\end{array}\right)\left(\alpha z^{p}\right)_{k}+\sum_{k=1}^{q+1}\left(\begin{array}{c}
\nu \\
k-1
\end{array}\right)\left(\beta z^{q+1}\right)_{k-1}\right] \phi_{\mu-k}(z)
$$




$$
\begin{gathered}
+\beta\left(\begin{array}{c}
\nu \\
q+1
\end{array}\right)(q+1) ! \phi_{\mu-q-2}(z)=f(z) \\
\left(\mu, \nu \in \mathbb{R} ; \alpha \neq 0 ; \beta \neq 0 ; p \in \mathbb{N} ; q \in \mathbb{N}_{0}\right)
\end{gathered}
$$

has a particular solution of the form:

$$
\begin{gathered}
\phi(z)=\left(\left(\frac{f_{-\nu}(z)}{\alpha z^{p}} e^{H(z ; p, q+1)}\right)_{-1} e^{-H(z ; p, q+1)}\right)_{\nu-\mu+1} \\
\left(z \in \mathbb{C} \backslash\left\{z: z^{p}=0(p \in \mathbb{N})\right\}\right),
\end{gathered}
$$

where $H(z ; p, q+1)$ is given by (2.13), it being provided that the second member of (2.15) exists.

Furthermore, the homogeneous linear ordinary fractional differintegral equation:

$$
\begin{gathered}
\alpha z^{p} \phi_{\mu}(z)+\left[\sum_{k=1}^{p}\left(\begin{array}{l}
\nu \\
k
\end{array}\right)\left(\alpha z^{p}\right)_{k}+\sum_{k=1}^{q+1}\left(\begin{array}{c}
\nu \\
k-1
\end{array}\right)\left(\beta z^{q+1}\right)_{k-1}\right] \phi_{\mu-k}(z) \\
+\beta\left(\begin{array}{c}
\nu \\
q+1
\end{array}\right)(q+1) ! \phi_{\mu-q-2}(z)=0 \\
\left(\mu, \nu \in \mathbb{R} ; \alpha \neq 0 ; \beta \neq 0 ; p \in \mathbb{N} ; q \in \mathbb{N}_{0}\right)
\end{gathered}
$$

has solutions of the form:

$$
\phi(z)=K\left(e^{-H(z ; p, q+1)}\right)_{\nu-\mu+1}
$$

where $K$ is an arbitrary constant and $H(z ; p, q+1)$ is given by (2.12) or (2.13), it being provided that the second member of (2.17) exists.

Remark 3. The function $H(z ; p, q+1)$ given by (2.13) would further simplify considerably if (for example) $\beta=-2 \alpha$, and we thus find from (2.13) that

$$
H(z ; p, q+1)=\left\{\begin{array}{cc}
-\frac{2 z^{q-p+2}}{q-p+2} & (p \neq q+2) \\
-2 \log z & (p=q+2) .
\end{array}\right.
$$




\section{Further Deductions from Theorem 2}

In the preceding section, we have already shown how readily Theorem 2 would follow as a special case of Theorem 1 (of Tu et al. [6]). Theorem 2, in turn, yields Theorem A (and hence also Theorem B) of Section 1 as one of its many further special cases. Indeed, in view of Lemma 1 and the case $p \neq q+2$ in (2.13), in its special case when

$$
\mu=m(m \in \mathbb{Z}), \nu=\frac{1}{2}(1-\lambda), p=q=n\left(n \in \mathbb{N}_{0}\right), \alpha=1, \text { and } \beta=-2,
$$

Theorem 2 (with, if relevant, $f(z)$ replaced trivially by $f(z) e^{\frac{1}{2} z^{2}}$ ) immediately yields Theorem A. And, just as we remarked in Section 1, Theorem B follows from Theorem A under the obvious notational change exhibited in (1.21).

\section{ACKNowledgments}

The present investigation was initiated during the fourth-named author's visits to Chung Yuan Christian University at Chung-Li and the Institute of Mathematics (Academia Sinica) at Nankang (Taipei) in August 1999. This work was supported, in part, by the National Science Council of the Republic of China under Grant NSC 89-2115-M-146-001 and the Natural Sciences and Engineering Research Council of Canada under Grant OGP0007353.

\section{REFERENCES}

1. K. Nishimoto, Fractional Calculus, Vols. I, II, III, and IV, Descartes Press, Koriyama, 1984, 1987, 1989, and 1991.

2. K. Nishimoto, An Essence of Nishimoto's Fractional Calculus (Calculus of the 21st Century) : Integrations and Differentiations of Arbitrary Order, Descartes Press, Koriyama, 1991.

3. I. Podlubny, Fractional Differential Equations, Mathematics in Science and Engineering, Vol. 198, Academic Press, New York, London, Tokyo, and Toronto, 1999.

4. H. M. Srivastava, S. Owa and K. Nishimoto, Some fractional differintegral equations, J. Math. Anal. Appl. 106 (1985), 360-366.

5. S.-T. Tu, Yu-Tan Huang and I-C. Chen, Generalized Weber equations via fractional calculus, Chung Yuan J. 28 (2000), 1-5.

6. S.-T. Tu, D.-K. Chyan and H. M. Srivastava, Some families of ordinary and partial fractional differintegral equations, Integral Transform. Spec. Funct. 9 (2000). 
Shih-Tong Tu, Yu-Tan Huang and I-Chun Chen Department of Mathematics, Chung Yuan Christian University

Chung-Li 32023, Taiwan, R.O.C.

E-mail: sttu@math.cycu.edu.tw

yutanhuang@yahoo.com

H. M. Srivastava

Department of Mathematics and Statistics University of Victoria

Victoria, British Columbia V8W 3P4, Canada

E-mail: harimsri@math.uvic.ca 